\title{
Charles Bonnet Syndrome Following Trans-Sphenoidal Adenomectomy without Optic Nerve Atrophy
}

\author{
Jang-Ho Park', Joon-Ho Ahn', Jun-Bum Park², and Soohyun Joe ${ }^{3 凶}$ \\ ${ }^{1}$ Department of Psychiatry, Ulsan University Hospital, University of Ulsan College of Medicine, Ulsan, Republic of Korea \\ ${ }^{2}$ Department of Neurosurgery, Ulsan University Hospital, University of Ulsan College of Medicine, Ulsan, Republic of Korea \\ ${ }^{3}$ Bugok National Hospital, Changnyeong, Republic of Korea
}

Charles Bonnet syndrome (CBS) can develop after trans-sphenoidal adenomectomy (TSA); however, the neural mechanisms remain unknown. Sensory deprivation and releasing phenomenon are both hypothetical explanations for this condition; however, there is no definite evidence that strongly supports either supposition. We report the first case of CBS after TSA without optic nerve atrophy. Postoperatively, the patient's vision seemed to be relatively well preserved, apart from the left-side hemianopsia in the right eye. Distinctive visual hallucinations only appeared when his eyes were closed, and these responded to quetiapine in a dose-dependent manner. Dose dependent change in colors and formation of hallucination was reported. Two weeks after quetiapine initiation, the patient's CBS was completely resolved. This unique case suggests that blocking sensory input from the periphery is more critical than neural damage of the bottom-up connection to the visual association cortex. In addition, quetiapine should be considered as a specific treatment for CBS.

Psychiatry Investig 2016;13(5):577-579

Key Words Charles Bonnet syndrome, Pathogenesis, Quetiapine, Trans-sphenoidal adenomectomy, Visual hallucination.

\section{INTRODUCTION}

The Charles Bonnet syndrome (CBS) was first described in 1760 , and it refers to visual hallucinations related to vision loss in patients with intact insight. ${ }^{1,2}$ Although it can be caused by abnormalities in the central or peripheral visual pathway, the most common clinical condition related to CBS is sensory deprivation due to vision loss (e.g. enucleation, eye patching, macular degeneration, etc.).

Two theories have been postulated regarding the origin of CBS: the release theory and the sensory deprivation theory., Both these theories are related to cortical disinhibition and spontaneous neuronal excitation of association cortices. In the release theory, a neural defect in the visual pathway causes abnormal signal transmission from the sensory end organ to the visual cortex. These false signals add to normal visual cor-

Received: August 11, 2015 Revised: October 26, 2015

Accepted: October 26, 2015 Available online: March 23, 2016

$\triangle$ Correspondence: Soohyun Joe, MD, PhD

Bugok National Hospital, 145 Bugok-ro, Bugok-myeon, Changnyeong 50365, Republic of Korea

Tel: +82-55-536-6440, Fax: +82-55-536-6444, E-mail: biophilialp@daum.net (a) This is an Open Access article distributed under the terms of the Creative Commons Attribution Non-Commercial License (http://creativecommons.org/licenses/bync/3.0) which permits unrestricted non-commercial use, distribution, and reproduction in any medium, provided the original work is properly cited. tical activity and these may result in complex visual hallucinations. ${ }^{3}$ In the sensory deprivation theory, the blocking of the sensory input facilitates the activation of the visual association cortex to generate hallucinations. ${ }^{4}$ Recently, failure of constraint on higher cortical function by reduced bottom-up sensory prediction was suggested as an explanation of phantom perception. However, given the rarity of the condition, there is a lack of convincing evidence in support of either theory.

CBS can occur as a rare complication of trans-sphenoidal adenomectomy (TSA). Just one case report describing postTSA CBS has been published, and vision loss due to adenomectomy-induced optic nerve atrophy was suggested to play an important role in CBS development. ${ }^{5}$

Our report describes the first case of CBS after TSA without optic nerve atrophy or vision loss. It provides the first evidence that sensory deprivation is required for the development of CBS, but neural damage to the bottom-up connection pathway to the visual association cortex is not.

\section{CASE}

A 46-year-old man underwent TSA for pituitary macroadenoma. Magnetic resonance imaging (MRI) was carried out before surgery (Figure 1A). Neurological examination was 
performed and the results showed limited lateral gaze of the right eye with diplopia.

On post-operative day (POD) 1 , the patient had intact insight and CBS first manifested. He experienced hallucinations when his eyes were closed, but they disappeared when he opened them. At the time, he was in the intensive care unit and kept his eyes closed for the majority of the time due to diplopia. The first hallucination was a black and white image of a giant sea anemone. The creature's tentacles were moving regularly and slowly. The hallucinations were so vivid that he first tried to catch them with his hands. He also reported seeing colorful animated images such as a desert, sculptures, fishes, curtains, and flower-like forms. At first, he did not complain because he enjoyed watching the phenomena. Only on POD 2 did he report that he could not fall asleep because of the hallucinations he saw when his eyes were closed. Ten milligram of Zolpidem was administered on that night but it was not effective. Alprazolam $0.25 \mathrm{mg}$ and clonazepam $0.5 \mathrm{mg}$ was tried on post-operative day 3 and also they were not effective. From post-operative day 4 to post-operative day 6,1 $\mathrm{mg} /$ day of lorazepam was tried but they were not effective in preventing the hallucinations.

Besides CBS reported during a psychiatric examination on POD 7, neurologic and mental status exams revealed no abnormalities. The patient did not show any evidence of cognitive impairment or consciousness fluctuation or disorientation. His Brief Psychiatric Rating Scale (BPRS) score was 24 (symptoms not present), and his Hamilton Depression Rating Scale (HDRS) score was 2.

On POD 8, $50 \mathrm{mg}$ of Quetiapine treatment was initiated, and the dose was increased to $200 \mathrm{mg}$ from POD 8 to POD 12. The patient reported that the colors of the hallucinations had attenuated since quetiapine was initiated; they gradually weakened and finally became monotone.

The image type also changed from stereotyped, repetitive, and animated figures to static ones. The decrease in CBS symptoms allowed him to sleep, and he reported being less tired. The patient's visual hallucinations gradually dissipated from POD 11 to 15 . On post-operative day 17, he was discharged with prescription of daily $200 \mathrm{mg}$ of quetiapine. When he visit outpatient psychiatric clinic at 2 weeks after discharge, his hallucinations were completely resolved. He was still suffering from diplopia and headache but he was on recovery. Quetiapine was reduced to $100 \mathrm{mg} /$ day until next visit at 6 weeks after discharge. The dose of quetiapine was gradually lowered and the treatment was eventually discontinued over the next 2 months, without the recurrence of hallucinations. His visual acuity and field were relatively well preserved postoperatively. However, limited lateralization of the right eyeball movement persisted until 2 months after surgery. His BPRS and HDRS scores 4 months after surgery were 24 and 0 , respectively. Follow-up MRI showed no abnormalities, apart from the expected post-operative changes of a cavity in the sella turcica and left-sided deviation of a mildly thickened pituitary stalk (Figure 1B). Last psychiatric follow up was 16 weeks after discharge and his BPRS and HDRS score wasn't changed Also he didn't wear eye patch anymore and his diplopia was nearly recovered.

\section{DISCUSSION}

There are no standardized criteria for the diagnosis of CBS; however, the following symptoms are considered: 1) persistent or repetitive complex visual hallucinations, 2) insight into
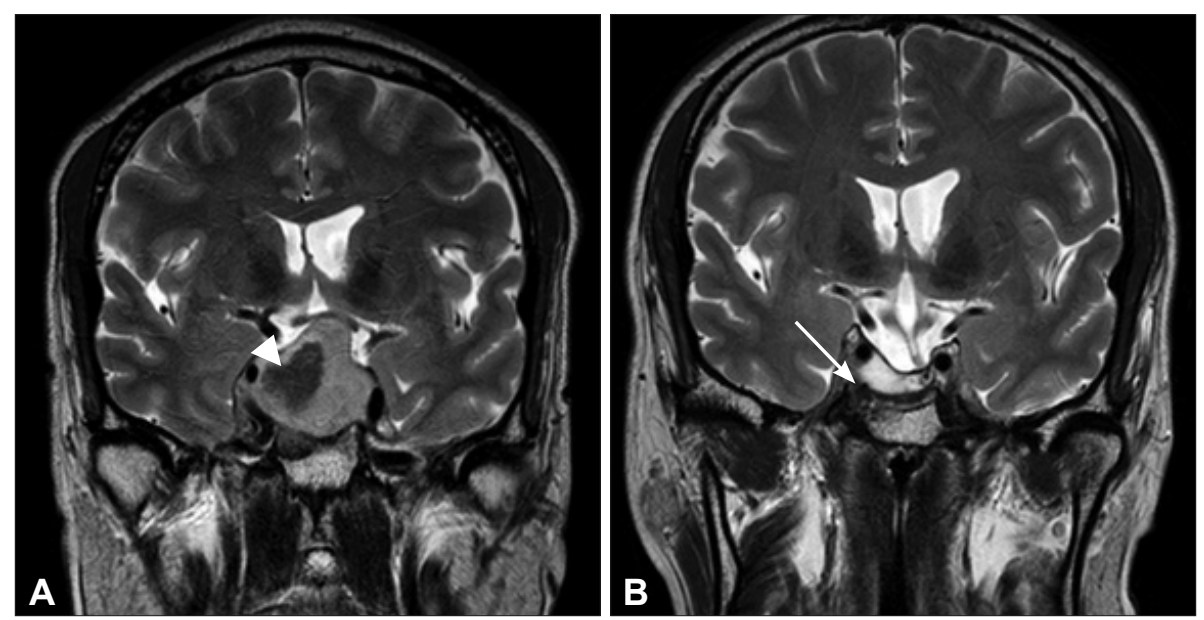

Figure 1. Brain MR images. A: Large cystic mass (arrowhead, $\sim 3.5 \mathrm{~cm}$ ) with mild focal wall enhancement and severe sellar floor widening. Suprasellar tumor extension with mild compression of the left optic chiasm, invasion of the right cavernous sinus, and encasement of the right cavernous internal carotid artery. Focal enhancing remnant gland at the left superior margin of the sellar mass. Pituitary macroadenoma with extensive cystic changes. B: After trans-sphenoidal adenomectomy to treat pituitary apoplexy and macroadenoma. The image reveals a $2.4 \times 0.8-\mathrm{cm}$ necrotic cavity without tumor remnants in the sella turcica (white arrow) and left-side deviation of the pituitary stalk with mild thickening. 
the hallucinations, 3) no other types of hallucinations, and 4) no primary or secondary delusions. ${ }^{1}$ The present patient met all four criteria, and no other psychiatric disorder was suspected following an interview with a psychiatrist. Differential diagnoses of delirium, dementia, Parkinsonism, epilepsy, and hypnagogic/hypnopompic hallucinations were excluded by psychiatric and neurologic exams.

So far, the pathophysiology of CBS has not been elucidated. Researchers have hypothesized that defects in the neural pathway from the optic nerve to the visual association cortex or sensory deprivation can cause spontaneous neuronal firing in the association cortex, thus facilitating the development of CBS. ${ }^{3,4}$ However, there is no sufficient evidence in support of either theory.

Interestingly, CBS in the present case occurred without vision loss due to optic atrophy. This, in itself, supports the sensory deprivation theory. His visual acuity and visual field were relatively well preserved during CBS; therefore, his visual pathway damage was not significant. Instead, CBS was induced when the patient blocked visual perception by closing his eyes. Simultaneous neural defects and sensory deficits were reported in previous cases related to TSA. In one case, the patient's vision was lost due to optic nerve atrophy after TSA. ${ }^{5}$ Another recent case report demonstrated that CBS can be the first symptom of pituitary adenoma with progressive bilateral vision loss due to optic nerve compression; however, this patient's symptoms improved after TSA. ${ }^{6}$ Other reports have claimed that most cases of CBS are associated with bilateral vision loss and that symptoms appear and disappear when the eyes are opened and closed, respectively. ${ }^{7.8}$ This is in direct opposition to the symptoms seen in our patient; CBS manifested when the patient's eyes were closed and disappeared upon eye opening. This observation supports the sensory deprivation theory of CBS. Although optic nerve damage due to an adenoma can induce CBS, that was not the case in the present patient, who maintained sensory input.
CBS is under-recognized because of low awareness among clinicians and low reporting by patients. ${ }^{2,7-9}$ The symptoms may cause the patient considerable distress and can persist for years. A recent study reported that $75 \%$ of CBS cases lasted for more than 5 years, and $32 \%$ had negative outcomes. Moreover, no specific treatment for CBS has been established. ${ }^{10}$ Among antipsychotics, olanzapine, risperidone, and quetiapine have been prescribed, and one report described that the combination of quetiapine and paroxetine was beneficial. Our experience suggests that quetiapine monotherapy can be a specific treatment for CBS. The response in the present case was dramatic and possibly dose-dependent.

\section{REFERENCES}

1. Gold K, Rabins PV. Isolated visual hallucinations and the Charles Bonnet syndrome: a review of the literature and presentation of six cases. Compr Psychiatry 1989;30:90-98.

2. Schadlu AP, Schadlu R, Shepherd JB 3rd. Charles Bonnet syndrome: a review. Curr Opin Ophthalmol 2009;20:219-222.

3. Khan JC, Shahid H, Thurlby DA, Yates JR, Moore AT. Charles Bonnet syndrome in age-related macular degeneration: the nature and frequency of images in subjects with end-stage disease. Ophthalmic Epidemiol 2008;15:202-208.

4. Menon GJ, Rahman I, Menon SJ, Dutton GN. Complex visual hallucinations in the visually impaired: the Charles Bonnet Syndrome. Surv Ophthalmol 2003;48:58-72.

5. Unsalver BO, Ozmen M, Velet S. Charles-Bonnet Syndrome: a report of two cases. Turk Psikiyatri Derg 2007;18:277-281.

6. Hashemi N, Zhang J, Gelman R, Lee AG. Visual hallucinations (Charles Bonnet syndrome) as the presenting sign of pituitary adenoma. Can J Ophthalmol 2012;47:e5-e6.

7. Brown GC, Murphy RP. Visual symptoms associated with choroidal neovascularization. Photopsias and the Charles Bonnet syndrome. Arch Ophthalmol 1992;110:1251-1256.

8. Cumurcu T, Elbozan Cumurcu B, Cam Celikel F. Charles Bonnet syndrome: a case presentation. Turk Psikiyatri Derg 2005;16:60-63.

9. Teunisse RJ, Cruysberg JR, Hoefnagels WH, van 't Hof MA, Verbeek AL, Zitman FG. Risk indicators for the Charles Bonnet syndrome. J Nerv Ment Dis 1998;186:190-192.

10. Cox TM, ffytche DH. Negative outcome Charles Bonnet syndrome. Br J Ophthalmol 2014;98:1236-1239. 